\title{
An intronic polymorphism of NFATC1 gene shows a risk association with biopsy-proven acute rejection in renal transplant recipients
}

\author{
Zijie Wang ${ }^{1 \#}$, Hengcheng Zhang ${ }^{1 \#}$, Haiwei Yang ${ }^{1 \#}$, Ming Zheng ${ }^{1}$, Miao Guo ${ }^{2}$, Hao Chen ${ }^{1}$, Li Sun ${ }^{1}$, \\ Zhijian Han ${ }^{1}$, Jun Tao ${ }^{1}$, Xiaobing Ju${ }^{1}$, Ruoyun Tan ${ }^{1}$, Ji-Fu Wei ${ }^{2}$, Min Gu ${ }^{1}$ \\ ${ }^{1}$ Department of Urology, ${ }^{2}$ Research Division of Clinical Pharmacology, The First Affiliated Hospital of Nanjing Medical University, Nanjing 210029 , \\ China \\ Contributions: (I) Conception and design: Z Wang, M Zheng, R Tan, M Gu; (II) Administrative support: H Zhang; (III) Provision of study materials \\ or patients: H Yang, M Guo, L Sun, Z Han, J Tao, JF Wei; (IV) Collection and assembly of data: H Zhang, H Yang, H Chen, X Ju; (V) Data analysis \\ and interpretation: Z Wang, R Tan, JF Wei, M Gu; (VI) Manuscript writing: All authors; (VII) Final approval of manuscript: All authors. \\ \#These authors contributed equally to this work. \\ Correspondence to: Ruoyun Tan, PhD; Min Gu, PhD. Department of Urology, The First Affiliated Hospital of Nanjing Medical University, Nanjing \\ 210029, China. Email: tanruoyun112@vip.sina.com; lancetgu@aliyun.com; Ji-Fu Wei, PhD. Research Division of Clinical Pharmacology, The First \\ Affiliated Hospital of Nanjing Medical University, Nanjing 210029, China. Email: weijifu@hotmail.com.
}

Background: We aimed to explore the influence of single nucleotide polymorphisms (SNPs) in NFATC1 gene on the occurrence of biopsy-proven acute rejection (BPAR) in renal transplant recipients.

Methods: Blood samples from 131 subjects with stable allograft function (STA) and 69 with BPAR episodes were collected and analyzed using target sequencing (TS) with an established panel. Odds ratios (OR) and 95\% confidence intervals (95\% CIs) were calculated for logistic regression models adjusted for confounding factors. Pathological changes were extracted and the relationship with tagger SNPs was calculated. Moreover, the CCK-8 assay was performed to explore the proliferation of T lymphocytes, and PCR, Western blotting and enzyme-linked immunosorbent assay were applied to identify the effect of mutant on the activation of $\mathrm{T}$ cells.

Results: High-quality readouts were obtained for 55 NFATC1 SNPs and 14 tagger SNPs were remained for further analysis. After adjusting for clinical confounding factors, the distribution of four NFATC1 SNPs, including rs2290154, rs2304738, rs754093 and rs754096, were statistically significant between STA and BPAR groups. Pathological association analysis indicated one SNP, rs2290154, was significantly related with the Banff score and renal tubulitis. Our in vitro study suggested that NFATC1 rs2290154 mutant could remarkably promote the $\mathrm{T}$ cell proliferation, increase the transcription of NFATC1 mRNA and expression of NFATC1 protein, as well as the interleukin-2 (IL-2) secretion.

Conclusions: We reported the crucial association of NFATC1 gene with the occurrence of acute rejection (AR) episodes. Moreover, in vitro NFATC1 rs2290154 was significantly involved in the T lymphocytes activation and proliferation through increasing the translation of NFATC1 mRNA and expression of NFATC1 protein, along with the secretion of IL-2.

Keywords: NFATC1; biopsy-proven acute rejection (BPAR); single nucleotide polymorphisms (SNPs); kidney transplantation

Submitted Oct 14, 2019. Accepted for publication Dec 17, 2019.

doi: 10.21037/atm.2020.01.61

View this article at: http://dx.doi.org/10.21037/atm.2020.01.61 


\section{Introduction}

Kidney transplantation has been considered as the optimal therapy for patients with end-stage renal diseases (1). Benefiting from novel immunosuppressive agents, incidence of acute rejection (AR) following kidney transplantation has dramatically declined over the past decades; however, approximately $10-15 \%$ of recipients currently suffer from AR during their first year post-transplant, which still adversely contribute to the loss of graft function (2-4). Recently, immunological factors, especially $\mathrm{T}$ cell-mediated immune responses, are believed to be strongly associated with the pathogenesis of AR episodes $(5,6)$.

Nuclear factor of activated T cells (NFAT) was originally observed in the nuclear extracts from activated $\mathrm{T}$ cells and was reported to activate $T$ cells by binding to the interleukin-2 (IL-2) promoter (7). Moreover, with regard to the extensive administration of cyclosporine A (CsA) and tacrolimus (TAC), which function by inhibiting calcineurin activity and NFAT nuclear translocation, as well as the consequent target gene transcription, the role of NFAT has been considered essential in the context of solid organ transplantation (8). The NFAT family of transcription factor consists of five members (NFATC1-NFATC5), which are all coded by different genes (9). In addition to NFATC5, which is activated in response to osmotic stress, all other NFAT proteins are regulated by the calcium and calcineurin signaling pathways (10-12). The calcium-related NFAT proteins consist of two conserved domains: (I) the Relhomology region (RHR); and (II) the NFAT homology region (NHR), which is a regulatory domain containing a calcineurin-binding site that regulates calcium, as well as calcium and calcineurin signaling pathways $(13,14)$. Moreover, the NHR is highly phosphorylated under steadystate condition, maintaining NFAT in an inactive state and restricted to the cytoplasm; NHR can be dephosphorylated under conditions of increased intracellular calcium levels and the activation of calcineurin, leading to NFAT cytoplasmic-nuclear trafficking $(15,16)$. Once in the nucleus, NFAT proteins function as transcription factors by binding to their target promoter regions (12).

The NFATC1 proteins are encoded by NFATC1 gene and located on chromosome $18 \mathrm{q} 23$. In addition, mice lacking the NFATC1 protein develop splenomegaly associated with the hyperproliferation of both $\mathrm{B}$ and $\mathrm{T}$ cells, combined with altered levels of IL-4, IL-13, and granulocyte-monocyte colony stimulating factor (GMCSF), indicating an essential role of NFATC1 on immune cell growth and transcription regulation (17). In solid organ transplantation, the regulation of gene expression (e.g., IL-2 and GM-CSF) by NFATC1 has been correlated with an over-immunosuppressive status, and is also observed in recipients who receive calcineurin inhibitors (CNIs) (18-20). While these studies illustrate the extensive relationship between NFATC1 proteins and the immune response, no studies have explored its influence on the AR episodes that occur following solid organ transplantation.

In this study, we performed a comprehensive analysis of NFATC1 single nuclear polymorphisms (SNPs) in renal transplant recipients using target sequencing (TS) based on next-generation sequencing (NGS), and 55 SNPs were detected. Then, 14 tagger SNPs were remained for further association analysis after the Hardy-Weinberg equilibrium (HWE) and major allele frequencies (MAF) analysis. The multivariable logistic regression analysis adjusted by two confounding clinical characteristics identified one significant SNP (rs2290154) with the remarkable pathological changes. Finally, our in vitro study transfected the mutated plasmid of rs2290154 and demonstrated that the mutation of rs2290154 promoted T cell proliferation by causing the increasingly translation of NFATC1 mRNA and synthesis of NFATC1 protein, which leads to the increased secretion of IL-2.

\section{Methods}

\section{Ethics approval and consent to participate}

Local ethics committee of the First Affiliated Hospital with Nanjing Medical University approved the protocols followed in this study (2016-SR-029). Written informed consents were obtained from all transplant recipients. All procedures performed in studies involving human participants were in accordance with the ethical standards of the institutional and/or national research committee and with the 1964 Helsinki declaration and its later amendments or comparable ethical standards. Recipients in this study was strictly limited to living-related transplantation of donors to lineal or collateral relatives not beyond the third degree of kinship or transplantation of kidney donors after cardiac death from 2011 to 2015.

\section{Patients and immunosuppressive therapy}

A total of 200 cases were selected among recipients who underwent kidney transplantation surgery between 
1st February 2011 and 1st December 2015 at the renal transplant center of First Affiliated Hospital with Nanjing Medical University. Detailed medical records and materials, including age, gender, transplant date, duration of transplant, transplant times, immunosuppressive protocol, panel reactive antibody (PRA) and human leukocyte antigen (HLA) mismatch, were carefully extracted by two independent clinicians (Zijie Wang and Ruoyun Tan) for patient selection.

Detailed immunosuppressive protocol following renal transplant was presented in our previous study (21). Briefly, all recipients received a three- or four-drug immunosuppressive regimen consisting of CsA or TAC in combination with mycophenolate mofetil (MMF) and prednisone (Pred), with or without sirolimus. The dosage of CsA and TAC was started at 8 and $0.2 \mathrm{mg} / \mathrm{kg} / \mathrm{d}$, respectively, and adjusted according to the results of therapeutic drug monitoring of the serum creatinine levels. A dosage of $200 \mathrm{mg} / \mathrm{d}$ of intravenous methylprednisolone was adopted for each BPAR episode lasting 3 to 5 days. The BPAR diagnostic criteria were based on the comprehensive evaluation of histological and clinical characteristics. The clinical characteristics were comprised of a $20 \%$ increase in serum creatinine levels from baseline (not attributable to other cases), fever, urine protein, and pain in the region of the transplanted kidney; histologically, the BPAR diagnosis was confirmed by an allograft biopsy according to Banff 07 classification (22), and consequently, the $\mathrm{i}, \mathrm{g}, \mathrm{t}, \mathrm{v}$, and ptc scores for each case were collected, which represent the interstitial inflammation, glomerulitis, tubulitis, intimal arteritis, and peritubular capillaritis, respectively.

During the follow-up of 3 years post-transplant for each subject, recipients who experienced at least one BPAR episode were assigned to BPAR group, while recipients with stable allograft function (STA) during the follow-up were enrolled to stable control group.

\section{Sample collection, preparation, and sequencing}

The detailed procedures of sample collection and the TS steps were described in our previous study (23). In brief, peripheral blood samples $(2 \mathrm{~mL})$ were collected from each recipient included in our study and the DNA was extracted. Quantitative detection of the concentration and purity of genomic DNA (gDNA) was performed while the gene integrity was tested by agarose gel electrophoresis. Next, a pool containing upstream and downstream oligonucleotides specific to the targeted regions of interest was hybrids to the gDNA samples. Quantitative detection was performed and fragmentation was followed by end repair, dA tailing, and sequencing adaptor ligation with an ABI 9700 PCR instrument (ABI, USA). The adapter-ligated DNA was amplified by selective, limited-cycle PCR and then quantitatively analyzed. The prepared library $(750 \mathrm{ng})$ was hybridized overnight and hybridized products were mixed with $200 \mu \mathrm{L}$ MyOne Streptavidin T1 magnetic beads (Invitrogen, USA) for $30 \mathrm{~min}$ at room temperature. Mixture was amplified for 16 PCR cycles and quantitatively assessed. The captured libraries were denatured and loaded onto an Illumina cBot instrument at 12 to $16 \mathrm{pmol} / \mathrm{L}$ for cluster generation according to the manufacturer's instructions. Up to $20 \mathrm{WUCaMP}$ libraries were sequenced per HiSeq lane.

\section{Data analysis}

Sequencing data, including the number of altered chromosomes, genomic alternation information, and the depth of sequencing coverage were analyzed. All analyses were based on the human reference sequence UCSC build hg19 (NCBI build 37.2) using the Burrows-Wheeler Aligner (BWA) (24). Local alignment and duplication removal were completed by the application of the Genome Analysis Tool Kit (GATK) and Picard software. SNP detection was performed using dbSNP 132. Damaging or deleterious SNPs were predicted using Gemini software. Prediction tools, including sorting intolerant from tolerant (SIFT) and polymorphism phenotyping (PolyPhen), were used to analyze all human non-synonymous SNPs. In addition, putative somatic variant calls were detected with two separate programs (MuTect 1.1.5 and VarScan 2.3.6 software) by pairing each sample with its matched blood.

\section{Cell culture}

The Jurkat $\mathrm{T}$ cell was obtained from the Type Culture Collection of the Chinese Academy of Sciences (Shanghai, China) and cultured in 1640 medium supplemented with $10 \%$ fetal bovine serum (FBS, Gibco, Australia) and 1\% penicillin-streptomycin in an incubator with humidified 5\% $\mathrm{CO}_{2}$ at $37^{\circ} \mathrm{C}$. The plasmids pCDNA3.1, pCMV-NFATC1 and pCMV-NFATC1-S617 were synthesized by Genechem (Shanghai, China) and then the transfection was performed according to the manufacturer's protocol. Detailed information of plasmids construction was presented in the Table S1. The cells were stimulated by PMA/Ionomycin mixture (Multi Sciences, Hangzhou, China) for $24 \mathrm{~h}$ among Stimulated group, pCDNA3.1 group, pCMV-NFATC1 
group and pCMV-NFATC1-S617 group. The naive Jurkat $\mathrm{T}$ cell was defined as Control group.

\section{RNA isolation and quantitative real-time PCR (qRT-PCR)}

Total RNA was extracted from cultured cells by using RNA extraction kits (Tiangen, China) according to the manufacture's protocol. Aliquots of total RNA were subjected to the RT reaction using PrimeScript RT reagent Kit (Takara, Japan). After reverse transcription, qRT-PCR were performed in triplicate in a 96-well plate containing $1 \mu \mathrm{L}$ of synthesized cDNA, QuantiNova ${ }^{\mathrm{TM}}$ SYBR Green PCR Kit (Qiagen, Germany) using StepOnePlus ${ }^{\mathrm{TM}}$ Real-Time PCR System (Applied Biosystems, USA) according to the manufacturer's instructions. The following primers were used for qRT-PCR: NFATC1, Forward: 5'-CCCACTCCTAGTTGCCACAT-3', Reverse: 5'- CCTGCTTTCCTTCTTGTTGG-3'). Fold changes in mRNA expression were calculated using $2^{-\Delta \Delta C t}$ method and normalized based on GADPH with ABI Step One Software version 2.1. All experiment was repeated for at least three times.

\section{Enzyme-linked immunosorbent assay (ELISA)}

ELISA kits (Multi Sciences, Hangzhou, China) were used to determine the supernatant levels of IL-2 in each group at $24 \mathrm{~h}$. The optical density (OD) value was measured at $450 \mathrm{~nm}$ on the microplate reader (Bio-Rad, USA). All procedures were followed strictly according to the manufacture's protocols. All experiment was repeated for at least three times.

\section{Cell proliferation assay}

The cells were seeded into 96-well plates, at a density of $1 \times 10^{4}$ cells per well, for the cell proliferation assay. Cell proliferation was detected using CCK-8 (Dojindo Molecular Technologies Inc., USA) following to the manufacture's protocols. The $10 \mu \mathrm{L}$ CCK- 8 was added to each well at the 24, 48, 72 and $96 \mathrm{~h}$ after stimulation, and then cultured for $4 \mathrm{~h}$. The OD values were read at $450 \mathrm{~nm}$ on the microplate reader (Bio-Rad, USA). All experiment was repeated for at least three times.

\section{Western blotting}

The protein of cells was extracted using RIPA buffer (Beyotime, China) with PMSF, and quantified by the BCA Protein Assay Kit according to the manufacturer's instructions (Beyotime,
China). Protein samples were separated by $10 \%$ SDS-PAGE gels, and then transferred onto polyvinylidene fluoride (PVDF) membranes. Following blocking with 5\% skimmed milk for $2 \mathrm{~h}$ at $24{ }^{\circ} \mathrm{C}$, the PVDF membranes were incubated with rabbit antibody specific to human NFAT1 (\#5861, Cell Signaling Technology, MA, USA, 1:1,000), or GAPDH (\#5174, Cell Signaling Technology, MA, USA, 1:1,000), or FLAG (\#14793, Cell Signaling Technology, MA, USA, 1:1,000) for overnight at $4{ }^{\circ} \mathrm{C}$. The membranes were then incubated with anti-rabbit secondary antibody for $2 \mathrm{~h}$ at $24^{\circ} \mathrm{C}$ (Thermo Fisher Scientific Inc., USA, 1:5,000). Chemiluminescence system (BioRad, USA) and Image Lab Software (Bio-Rad Laboratories Inc., USA) were used to analyze the protein expression. All experiment was repeated for at least three times.

\section{Statistical analysis}

HWE and MAF analysis were performed using gene frequencies obtained by a single gene counting. We explored the linkage disequilibrium (LD) blocks and tagger SNPs using Haploview version 4.2 software. A genotype association analysis of tagger SNPs was performed using the dominant model (minor allele homozygotes plus heterozygotes $v s$. major allele homozygotes), recessive model (minor allele homozygotes $v s$. heterozygotes plus major homozygotes), additive model (major homozygotes $v s$. heterozygotes $v s$. minor homozygotes), HET model (major homozygotes $v s$. heterozygotes) and HOM model (major homozygotes vs. minor homozygotes). Comparisons of the genotypic frequencies between the STA and BPAR groups were assessed using a chi-square test. Genotypic distributions of tagger SNPs between two groups were compared with logistic regression model adjusted for confounding factors identified by general linear model (GLM). Then, significant tagger SNPs were included for further evaluation of the relationship with the pathological changes by using the multivariable logistic regression model. Odds ratios (OR) and $95 \%$ confidence intervals (95\% CIs) were calculated using SPSS 13.0 software (SPSS Inc., Chicago, IL, USA). Significant SNPs with BPAR episodes and pathological changes in recipients were selected for the in vitro function validation. Results were presented as mean \pm standard deviation (SD) from at least three independent experiments using GraphPad Prism 6.0 software (San Diego, CA, USA). Comparisons between mutation and wild-type groups were conducted using an unpaired Student's $t$-test or 2-way ANOVA. A threshold of $\mathrm{P}<0.05$ was considered significant. The Bonferroni correction 
Table 1 Basic characteristics of patients included in our cohort

\begin{tabular}{|c|c|c|c|}
\hline Variables & STA group & BPAR group & $P$ value \\
\hline Donor age (years; mean \pm SD) & $42.32 \pm 2.05$ & $40.76 \pm 3.09$ & NS \\
\hline Donor male (\%) & 61.09 & 57.97 & NS \\
\hline Recipient age (years; mean \pm SD) & $38.56 \pm 1.40$ & $38.92 \pm 1.02$ & NS \\
\hline Donor type & & & NS \\
\hline Living-related & 16 & 7 & \\
\hline DCD & 115 & 62 & \\
\hline Pre-transplant PRA (\%; mean \pm SD) & $0.80 \pm 0.46$ & $2.12 \pm 0.99$ & NS \\
\hline Pred + MMF + CsA & 39 & 31 & \\
\hline Pred + MMF + TAC & 87 & 19 & \\
\hline Pred + MMF + CsA + SIR & 3 & 3 & \\
\hline Pred + MMF + TAC + SIR & 2 & 16 & \\
\hline
\end{tabular}

BPAR, biopsy-proven acute rejection; STA, stable allograft function; NS, not significant; SD, standard deviation; DCD, donor after cardiac death; PRA, panel reactive antibody; Pred, prednisone; MMF, mycophenolate mofetil; CsA, cyclosporin A; TAC, tacrolimus; SIR, sirolimus.

method was performed to avoid inflation of $\mathrm{P}$ value when multiple comparisons happened.

\section{Results}

\section{Study population}

The baseline characteristics of two study groups are presented in Table 1. No significant differences associated with the donor age, donor gender, recipient age, and recipient gender were observed $(\mathrm{P}>0.05)$. Among all the enrolled recipients, the majority $(88.5 \%)$ received donor grafts from a donor after cardiac death (DCD). Although the mean levels of pre-transplant PRA and HLA mismatches, as well as the sirolimus usage proportion in the BPAR group were relatively higher than those in the STA group, no statistical differences were found $(\mathrm{P}>0.05)$.

\section{SNP identification}

A total of 55 SNPs located in the NFATC1 gene were identified by TS based on NGS in our study. The genetic information of all detected SNPs was presented in Table S2.
After HWE and MAF analysis, as well as the removal of SNPs with HWE less than 0.05 and/or minor allele frequencies (MAF <0.05), 20 SNPs were obtained (Table S3). Furthermore, 14 tagger SNPs were selected for further association analysis by LD analysis (Figure 1).

\section{Association of NFATC1 SNPs with AR episodes}

Next, GLM analysis identified two confounding factors, CsA/TAC usage and sirolimus administration (CsA/TAC usage: $\mathrm{t}=-2.50, \mathrm{P}=0.013$; sirolimus administration: $\mathrm{t}=4.79$, $\mathrm{P}<0.001$; Table 2). Then, a logistic regression analysis with an adjustment for CsA/TAC usage and sirolimus administration was performed for all five analytical models (dominant, recessive, additive, HET and HOM) to explore the relationship between NFATC1 SNPs and BPAR episodes following kidney transplantation. Overall, four SNPs in the NFATC1 gene (rs2290154, rs2304738, rs754093 and rs754096) were summarized to be statistically significant between STA and BPAR groups (Table 3). We did not observe any significant difference in other tagger SNPs (Table S4). In addition, four haplotypes were identified by LD analysis and there was no significant difference with 


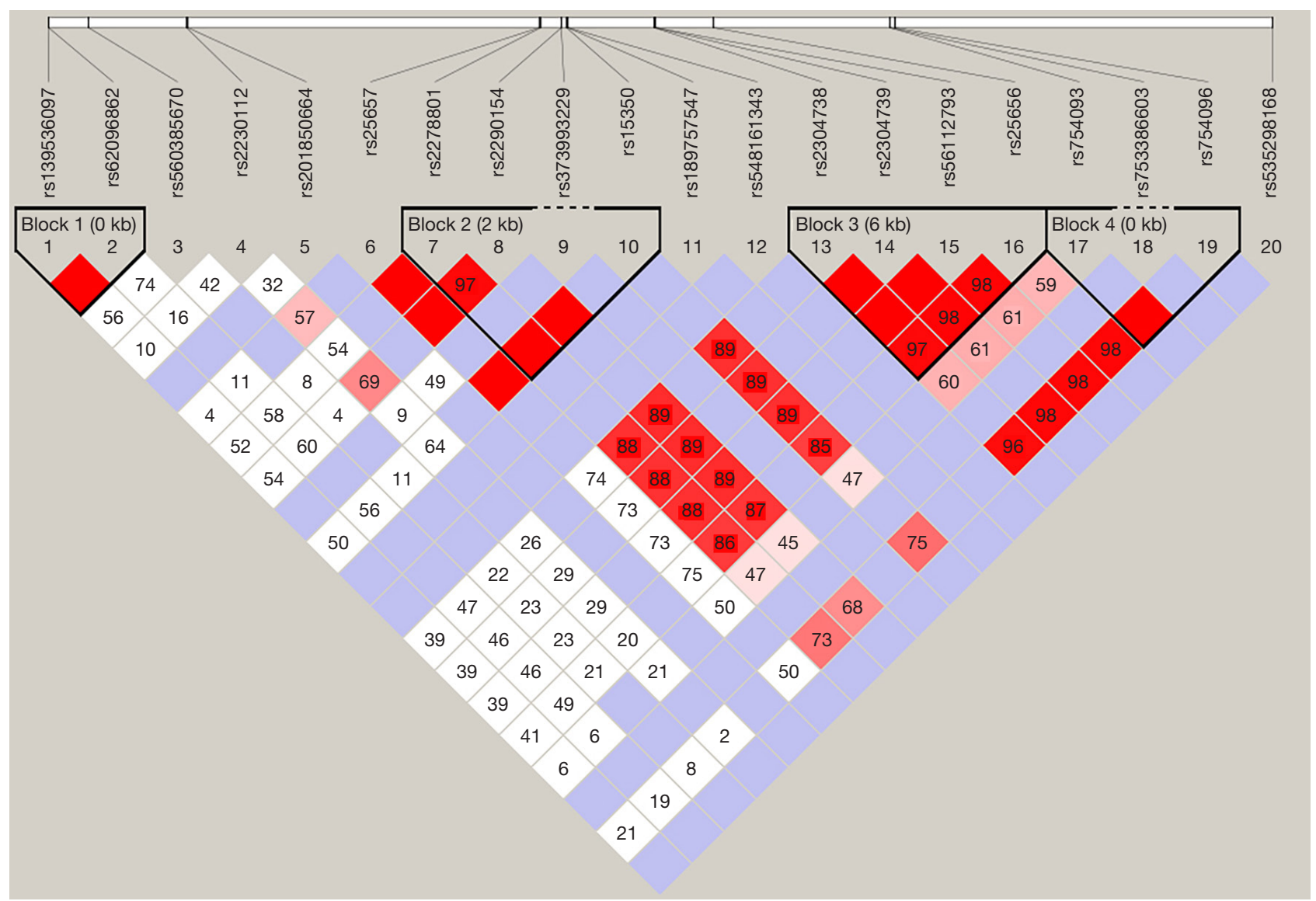

Figure 1 LD results of detected SNPs in the NFATC1 gene. LD, linkage disequilibrium; SNPs, single nucleotide polymorphisms.

Table 2 Results of clinical confounding factors identification analysis in this cohort using the GLM

\begin{tabular}{lccc}
\hline Variables & $\begin{array}{c}\text { Standardized } \\
\text { coefficients }\end{array}$ & t value & P value \\
\hline Age & -0.032 & -0.48 & 0.63 \\
Gender & 0.036 & 0.54 & 0.59 \\
CsATTAC usage & -0.17 & -2.50 & 0.013 \\
Transplant duration & 0.046 & 0.66 & 0.51 \\
Sirolimus administration & 0.33 & 4.79 & $<0.001$ \\
DGF episodes & 0.13 & 1.94 & 0.054 \\
\hline
\end{tabular}

CsA, cyclosporine A; TAC, tacrolimus; DGF, delayed graft function; GLM, general linear model.

BPAR episodes.

\section{Association of NFATC1 SNPs with pathological examination}

We examined all 69 recipients suffering from the BPAR episodes and reviewed the each case of pathological slides by two independent pathological professors (H Chen and RY Tan) according to Banff 07 criteria. The results of pathological evaluation were presented in Table 4. We explored the relationship between four significant SNPs and pathological changes, and found that only one SNP, rs2290154, was significantly associated with Banff score and $t$ score using multivariable logistic regression (Banff score: $\mathrm{t}=-3.12, \mathrm{P}=0.003$; $\mathrm{t}$ score: $\mathrm{t}=2.98, \mathrm{P}=0.004$; Table 5). However, no difference of other three SNPs (rs2304738, rs754093, and rs754096) was observed (Table S5).

\section{NFATC1 rs2290154 mutant promotes T cells proliferation and activation}

To identify the biological function of rs2290154 mutation on the NFATC1 gene, we transfected the wild-type and mutated plasmids of rs2290154 into T lymphocytes, respectively. We tested the cell proliferation and found 
Table 3 Results of association analysis of four significant taggers SNPs located on NFATC1 gene with post-transplant BPAR episodes using multivariable logistic regression analysis adjusted by two confounding factors (CsA/TAC usage and sirolimus administration)

\begin{tabular}{|c|c|c|c|c|}
\hline SNPS & Model & OR & $95 \% \mathrm{Cls}$ & $P$ value \\
\hline \multirow{3}{*}{ rs2290154 } & Dominant & 2.35 & $1.17-4.74$ & 0.02 \\
\hline & Recessive & 3.33 & $1.47-7.52$ & 0.003 \\
\hline & HOM & 1.87 & $0.90-3.90$ & 0.09 \\
\hline \multirow[t]{4}{*}{ rs2304738 } & Additive & 2.13 & $1.31-3.45$ & 0.002 \\
\hline & Dominant & 2.27 & $1.18-4.36$ & 0.01 \\
\hline & Recessive & 3.35 & $1.32-8.48$ & 0.01 \\
\hline & HOM & 1.89 & $0.96-3.75$ & 0.06 \\
\hline \multirow{4}{*}{ rs754093 } & Dominant & 2.54 & $1.29-4.97$ & 0.006 \\
\hline & Recessive & 3.64 & $1.60-8.27$ & 0.002 \\
\hline & $\mathrm{HOM}$ & 5.56 & $2.16-14.27$ & 0.0003 \\
\hline & HET & 1.98 & $0.98-4.03$ & 0.05 \\
\hline \multirow[t]{4}{*}{ rs754096 } & Additive & 2.58 & $1.59-4.22$ & 0.0001 \\
\hline & Dominant & 3.44 & $1.48-7.98$ & 0.004 \\
\hline & Recessive & 3.28 & $1.61-6.68$ & 0.001 \\
\hline & HOM & 6.72 & $2.48-18.14$ & 0.0001 \\
\hline
\end{tabular}

*, $P$ value less than adjusted $P$ value $(0.05 / 14=0.0036)$ was considered as statistical significant. BPAR, biopsy-proven acute rejection; SNPs, single nucleotide polymorphisms; CsA, cyclosporine A; TAC, tacrolimus; OR, odds ratio; Cls, confidential intervals.

Table 4 Pathological evaluation results of 69 recipients in BPAR group of the cohort

\begin{tabular}{lc}
\hline Variables* $^{*}$ & Results \\
\hline Case number & 69 \\
Banff score & $2.13 \pm 1.12$ \\
i score & $2.43 \pm 0.67$ \\
g score & 0 \\
t score & $1.84 \pm 0.68$ \\
v score & $0.22 \pm 0.51$ \\
ptc score & $1.06 \pm 0.66$ \\
\hline
\end{tabular}

*, data was presented as mean $\pm \mathrm{SD}$; ", Banff score was obtained according to the Banff 2007 classification. The i, g, t, v, and ptc scores represent the interstitial inflammation, glomerulitis, tubulitis, intimal arteritis, and peritubular capillaritis, respectively. BPAR, biopsy-proven acute rejection; SD, standard deviation.
Table 5 Results of association analysis of rs2290154 on NFATC1 gene with the pathological scores obtained from 69 recipients in BPAR group by logistic regression analysis

\begin{tabular}{lccl}
\hline Variables & $\begin{array}{c}\text { Standardized } \\
\text { coefficients }\end{array}$ & $\mathrm{t}$ & $\mathrm{P}$ value* \\
\hline i score & 0.10 & 0.77 & 0.44 \\
t score & -0.83 & -3.12 & 0.003 \\
v score & -0.54 & -2.05 & 0.045 \\
ptc score & 0.20 & 1.69 & 0.097 \\
Banff score & 1.14 & 2.98 & 0.004 \\
\hline
\end{tabular}

*, $P$ value less than adjusted $P$ value $(0.05 / 4=0.0125)$ was considered as statistical significant; " , Banff score was obtained according to the Banff 2007 classification. The i, g, t, v, and ptc scores represent the interstitial inflammation, glomerulitis, tubulitis, intimal arteritis, and peritubular capillaritis, respectively. BPAR, biopsy-proven acute rejection. 
A

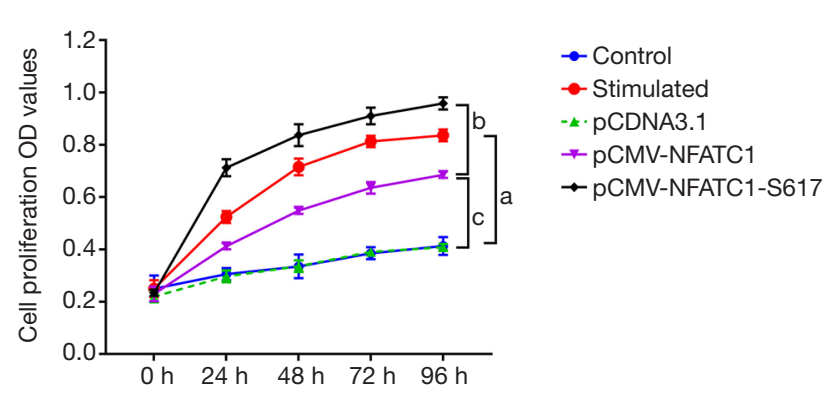

C

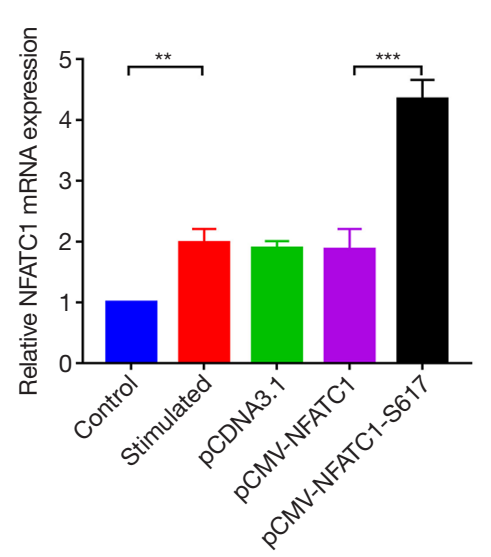

B
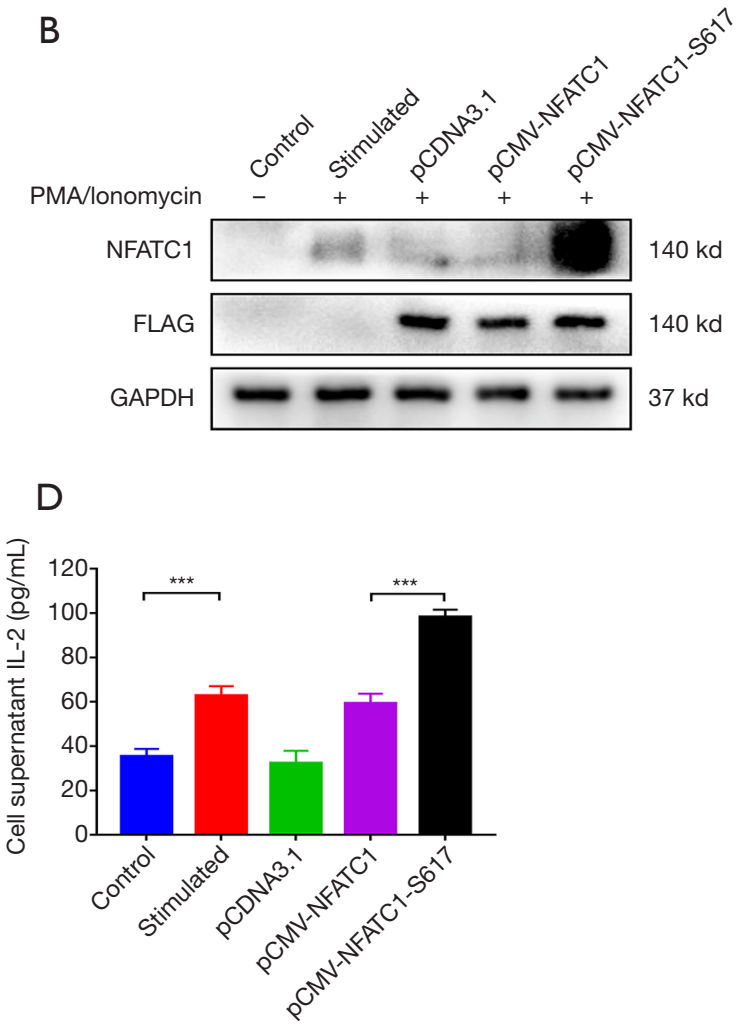

Figure 2 NFATC1 rs2290154 mutant promotes the T cells proliferation and activation in vitro. (A) We used NFATC1 rs2290154 wild-type and mutation plasmid to transfect the T cells in vitro and CCK-8 assay showed that NFATC1 rs2290154 mutant significantly promoted the $\mathrm{T}$ cells proliferation when compared with the wild-type group and stimulation group ( ${ }^{\mathrm{a}}, \mathrm{P}<0.001$, stimulated group vs. control group; b, $\mathrm{P}<0.001$, pCMV-NFATC1-S617 group vs. pCMV-NFATC1 group; ${ }^{\mathrm{c}}, \mathrm{P}<0.05$, pCMV-NFATC1 group $v s$. pCDNA3.1 group); (B) total proteins from each group were extracted and western blotting results demonstrated the significant increase of NFATC1 protein stimulated by NFATC1 rs2290154 mutant; (C) total DNA from each group was extracted and NFATC1 mRNA was tested by RT-PCR. Results indicated the significant increase of NFATC1 mRNA stimulated by NFATC1 rs2290154 mutant (data are means \pm SD; **, $\mathrm{P}<0.01$; ***, $\mathrm{P}<0.001$ ); (D) supernatant from cell culture in each group was collected and ELISA was used to examine the concentration of IL-2. We found the statistically increased secretion of IL-2 cytokines after the intervention of NFATC1 rs2290154 mutation plasmids (data are means \pm SD; ${ }^{* * *}$, $\mathrm{P}<0.001)$. RT-PCR, real-time PCR; SD, standard deviation; ELISA, enzyme-linked immunosorbent assay; IL-2, interleukin-2.

that NFATC1 rs2290154 mutant significantly promoted the proliferation of $\mathrm{T}$ lymphocytes, especially at 48 and $72 \mathrm{~h}$ after stimulation (Figure $2 A$ ). Then, we examined the translation of NFATC1 mRNA and expression of NFATC1 protein, the results showed that NFATC1 mRNA and NFATC1 protein was significantly increased by the NFATC1 rs2290154 mutant in T lymphocytes (Figure 2B,C), along with the remarkable increase of IL-2 in supernatant (Figure 2D).

\section{Discussion}

Renal transplant recipients are generally exposed to combined immunosuppressive therapy with a limited therapy window, which is associated with a higher rate of BPAR episodes. The NFATC1 gene was found to influence the susceptibility to AR by altering the activity and expression of NFATC1 protein, which plays an important role in gene transcription for mounting an immune response (25). Here, we conducted an association study of Chinese renal transplant recipients to comprehensively explore the effects of the NFATC1 gene on the pathogenesis of AR episodes. Sequencing study has identified one SNP, rs2290154, was significantly associated with the BPAR episodes and pathological changes, especially in the Banff score and renal tubulitis. Furthermore, our in vitro study has 
identified what we believe is a novel molecular mechanism by which NFATC1 rs2290154 mutant promoted the T lymphocytes activation and proliferation through increasing the translation of NFATC1 mRNA and expression of NFATC1 protein, and the secretion of IL-2, which is responsible for the increased risk of $\mathrm{AR}$ episodes following renal transplant.

It is well established that NFAT transcription factors play critical roles in CD4 $\mathrm{T}$ cell activation and differentiation and calcineurin/NFAT network has an important function in the generation and function of activated $\mathrm{T}$ lymphocytes, including CD4+ and CD8+ $\mathrm{T}$ cells $(26,27)$. In one study, a defective nuclear translocation of NFATC1 has been described for NFATC1 in CD8+ $\mathrm{T}$ cells, not in $\mathrm{CD} 4+\mathrm{T}$ cells (27). Consistently, ablation of NFATC1 results in an impaired formation of F-actin rings around the immunological synapse in cytotoxicity T lymphocyte (CTLs) upon TCR stimulation, indicating that NFATC1 controls the effector function of CD8+ T cells at multiple levels (26). To date, no association studies have been performed to explore the relationship between NFATC1 gene polymorphisms and the AR episodes that occur following kidney transplantation. Moreover, the mechanisms of such interactions remain unclear. In a clinical study performed in cases of human allograft rejection, an increase in calpain was observed in infiltrating $\mathrm{T}$ cells, providing novel prospects for pharmacological manipulation of the calpain/calpastatin balance during solid organ transplantation (28). Since calpain is a calciumactivated protease which is involved in the activation of NF$\kappa \mathrm{B}$ and NFAT, the outcomes in this study indicate that the NFAT gene may indirectly participate in the development of rejection. In our study, we sequenced the loci mutations on NFACT1 gene and identified one SNP mutant may have a positive impact on the NFATC1 protein expression, as well as the cell proliferation, explaining the results observed in our cohort. We provided the genetic evidence of NFATC1 in the pathogenesis of $\mathrm{AR}$ episodes following renal transplant, which could be used as a candidate gene for the regulation of massive T lymphocyte activation and proliferation.

Recently, published GWAS studies summarized that nearly $80 \%$ of trait-associated SNPs are located in non-coding regions, such as intron and untranslated region (UTR) (29). Results from numerous studies have implicated crucial regulatory functions to noncoding intronic loci on human genes (30,31). From our sequencing data and in vitro results, we have concluded that one intron SNP located in NFATC1, rs2290154, may contribute to the significant proliferation and activation of $\mathrm{T}$ lymphocytes in AR episodes by the mutation from $\mathrm{T}$ allele to $\mathrm{C}$ allele. Since no previous studies have associated this mutation with the function of $\mathrm{T}$ cells, we were unable to explain the detailed regulatory mechanism of rs2290154 mutant in AR episodes. Furthermore, we have simultaneously verified that two SNPs, rs25657 and rs2278801, in linkage with rs2290154 were not in the chromatin regions annotated as promoters and enhancers. Taken together, our results suggest that the NFATC1 gene may be a plausible candidate gene for the pathogenesis of post-transplantation $\mathrm{AR}$ episodes by encoding a transcription factor (rs2290154 mutant) which regulates gene transcription during an immune response (32).

Some limitations associated with our study should also be acknowledged: (I) this study contained a limited number of subjects from a single transplant center, which inevitably led to the limited power to detect a significant association. Thus, the results needs to be validated by replication in different patient cohorts; (II) there was a lack of information pertaining to the exposure to environmental factors in this study (e.g., diet and lifestyle); (III) our in vitro study reveals the positive regulation of NFATC1 rs2290154 mutant on $\mathrm{T}$ cells proliferation, which partly explained the results observed in the occurrence of BPAR episodes of this cohort; and (IV) with regard to the complexity of AR episodes following kidney transplantation, various genetic and non-genetic factors have been identified to partially contribute to the BPAR; while in our study, mainly the genetic factors were explored with the lack of non-genetic factors investigation, which should be interpreted with great caution. However, extensive explore to the influence of this mutant on the $\mathrm{T}$ cell activation, differentiation and metabolism are still warrant.

In summary, our study firstly reported the crucial function of NFATC1 gene in the occurrence of AR episodes. Using the TS based on the NGS technology, we have identified one significant SNP, rs2290154, is significantly associated with the remarkable renal allograft pathological changes. Our in vitro study further observed that the NFATC1 rs2290154 mutant could significantly promoted the $\mathrm{T}$ cells proliferation, increased the translation of NFATC1 mRNA and the expression of NFATC1 protein, as well as the secretion of IL-2. Given the known trigger function of NFATC1 in T lymphocytes, these findings suggest a possible link between genetic variation in NFATC1 and the onset of AR following renal transplant. Future studies will be required to validate the importance of NFATC1 rs2290154 mutant in the progression of AR episodes. 


\section{Acknowledgments}

Funding: This work was supported by the National Natural Science Foundation of China (grant numbers 81900684, 81870512, 81770751, 81570676, 81470981, 81100532), Project of Jiangsu Province for Important Medical Talent (grant number ZDRCA2016025), the "333 High Level Talents Project" in Jiangsu Province (grant numbers BRA2017532, BRA2016514, BRA2015469), the Standardized Diagnosis and Treatment Research Program of Key Diseases in Jiangsu Province (grant number BE2016791), the Open Project Program of Health Department of Jiangsu Province (grant number JSY-22016-099), Jiangsu Province Natural Science Foundation Program (grant number BK20191063).

\section{Footnote}

Conflicts of Interest: The authors have no conflicts of interest to declare.

Ethical Statement: The authors are accountable for all aspects of the work in ensuring that questions related to the accuracy or integrity of any part of the work are appropriately investigated and resolved. Local ethics committee of the First Affiliated Hospital with Nanjing Medical University approved the protocols followed in this study (2016-SR-029). Written informed consents were obtained from all transplant recipients. All procedures performed in studies involving human participants were in accordance with the ethical standards of the institutional and/or national research committee and with the 1964 Helsinki declaration and its later amendments or comparable ethical standards.

Open Access Statement: This is an Open Access article distributed in accordance with the Creative Commons Attribution-NonCommercial-NoDerivs 4.0 International License (CC BY-NC-ND 4.0), which permits the noncommercial replication and distribution of the article with the strict proviso that no changes or edits are made and the original work is properly cited (including links to both the formal publication through the relevant DOI and the license). See: https://creativecommons.org/licenses/by-nc-nd/4.0/.

\section{References}

1. Wang Z, Han Z, Tao J, et al. Transforming growth factor- $\beta 1$ induces endothelial-to-mesenchymal transition via Akt signaling pathway in renal transplant recipients with chronic allograft dysfunction. Ann Transplant 2016;21:775-83.

2. Ekberg H, Tedesco-Silva H, Demirbas A, et al. Reduced exposure to calcineurin inhibitors in renal transplantation. N Engl J Med 2007;357:2562-75.

3. Cole EH, Johnston O, Rose CL, et al. Impact of acute rejection and new-onset diabetes on long-term transplant graft and patient survival. Clin J Am Soc Nephrol 2008;3:814-21.

4. Dunn TB, Noreen H, Gillingham K, et al. Revisiting traditional risk factors for rejection and graft loss after kidney transplantation. Am J Transplant 2011;11:2132-43.

5. Ayed K, Abdallah TB, Bardi R, et al. Plasma levels of soluble CD30 in kidney graft recipients as predictors of acute allograft rejection. Transplant Proc 2006;38:2300-2.

6. Hariharan S. Long-term kidney transplant survival. Am J Kidney Dis 2001;38:S44-50.

7. Shaw JP, Utz PJ, Durand DB, et al. Identification of a putative regulator of early $\mathrm{T}$ cell activation genes. Science 1988;241:202-5.

8. Liu J, Farmer JD Jr, Lane WS, et al. Calcineurin is a common target of cyclophilin-cyclosporin A and FKBPFK506 complexes. Cell 1991;66:807-15.

9. Xu Q, Qiu X, Jiao Z, et al. NFATC1 genotypes affect acute rejection and long-term graft function in cyclosporinetreated renal transplant recipients. Pharmacogenomics 2017;18:381-92.

10. López-Rodríguez C, Aramburu J, Jin L, et al. Bridging the NFAT and NF-kappaB families: NFAT5 dimerization regulates cytokine gene transcription in response to osmotic stress. Immunity 2001;15:47-58.

11. Rao A, Luo C, Hogan PG. Transcription factors of the NFAT family: regulation and function. Annu Rev Immunol 1997;15:707-47.

12. Hogan PG, Chen L, Nardone J, et al. Transcriptional regulation by calcium, calcineurin, and NFAT. Genes Dev 2003;17:2205-32.

13. Macian F. NFAT proteins: key regulators of T-cell development and function. Nat Rev Immunol 2005;5:472-84.

14. Lopez-Rodríguez C, Aramburu J, Rakeman AS, et al. NFAT5, a constitutively nuclear NFAT protein that does not cooperate with Fos and Jun. Proc Natl Acad Sci U S A 1999;96:7214-9.

15. Shibasaki F, Price ER, Milan D, et al. Role of kinases and the phosphatase calcineurin in the nuclear shuttling of 
transcription factor NF-AT4. Nature 1996;382:370-3.

16. Clipstone NA, Crabtree GR. Identification of calcineurin as a key signalling enzyme in T-lymphocyte activation. Nature 1992;357:695-7.

17. Hodge MR, Ranger AM, Charles de la Brousse F, et al. Hyperproliferation and dysregulation of IL-4 expression in NF-ATp-deficient mice. Immunity 1996;4:397-405.

18. Zahn A, Schott N, Hinz U, et al. Immunomonitoring of nuclear factor of activated $\mathrm{T}$ cells-regulated gene expression: the first clinical trial in liver allograft recipients. Liver Transpl 2011;17:466-73.

19. Sommerer C, Zeier M, Meuer S, et al. Individualized monitoring of nuclear factor of activated $\mathrm{T}$ cells-regulated gene expression in FK506-treated kidney transplant recipients. Transplantation 2010;89:1417-23.

20. Sommerer C, Zeier M, Czock D, et al. Pharmacodynamic disparities in tacrolimus-treated patients developing cytomegalus virus viremia. Ther Drug Monit 2011;33:373-9.

21. Wang Z, Zheng M, Yang H, et al. Association of genetic variants in CYP3A4, CYP3A5, CYP2C8, and CYP2C19 with tacrolimus pharmacokinetics in renal transplant recipients. Curr Drug Metab 2019;20:609-18.

22. Solez K, Colvin RB, Racusen LC, et al. Banff 07 classification of renal allograft pathology: updates and future directions. Am J Transplant 2008;8:753-60.

23. Wang Z, Yang H, Si S, et al. Polymorphisms of nucleotide factor of activated $T$ cells cytoplasmic 2 and 4 and the risk of acute rejection following kidney transplantation. World J Urol 2018;36:111-6.

Cite this article as: Wang $Z$, Zhang $H$, Yang $H$, Zheng $M$, Guo M, Chen H, Sun L, Han Z, Tao J, Ju X, Tan R, Wei JF, Gu M. An intronic polymorphism of NFATC1 gene shows a risk association with biopsy-proven acute rejection in renal transplant recipients. Ann Transl Med 2020;8(5):211. doi: 10.21037/atm.2020.01.61
24. Li H, Durbin R. Fast and accurate long-read alignment with Burrows-Wheeler transform. Bioinformatics 2010;26:589-95.

25. Peng SL, Gerth AJ, Ranger AM, et al. NFATc1 and NFATc2 together control both $\mathrm{T}$ and $\mathrm{B}$ cell activation and differentiation. Immunity 2001;14:13-20.

26. Klein-Hessling S, Muhammad K, Klein M, et al. NFATc1 controls the cytotoxicity of $\mathrm{CD}^{+} \mathrm{T}$ cells. Nat Commun 2017;8:511.

27. Leung-Theung-Long S, Mondor I, Guiraud M, et al. Impaired NFAT transcriptional activity in antigen-stimulated CD8 T cells linked to defective phosphorylation of NFAT transactivation domain. J Immunol 2009;182:6807-14.

28. Letavernier E, Dansou B, Lochner M, et al. Critical role of the calpain/calpastatin balance in acute allograft rejection. Eur J Immunol 2011;41:473-84.

29. Hindorff LA, Sethupathy P, Junkins HA, et al. Potential etiologic and functional implications of genome-wide association loci for human diseases and traits. Proc Natl Acad Sci U S A 2009;106:9362-7.

30. ENCODE Project Consortium. An integrated encyclopedia of DNA elements in the human genome. Nature 2012;489:57-74.

31. Gerstein MB, Kundaje A, Hariharan M, et al. Architecture of the human regulatory network derived from ENCODE data. Nature 2012;489:91-100.

32. Horsley V, Pavlath GK. NFAT: ubiquitous regulator of cell differentiation and adaptation. J Cell Biol 2002;156:771-4. 


\section{Supplementary}

Table S1 Detailed information of NFATC1 plasmids construction

Vector information

General: 10418bp

ORF frame 1: 1558-2445

EGFP: $4350-5069$

Ampicillin: 9425-10285

HIV-1_5_LTR, trunkHIV-1_3_LTR: 835-1015

HIV-1_5_LTR, trunkHIV-1_3_LTR: 6223-6403

CAG_enhancer: 318-605

Psi: 1067-1204

RRE: $1680-1913$

cРPT: 2444-2459

H1-F: 2479-2502

3FALG: $3880-3957$

WPRE: 5112-5699

pGC-E1-SEQR: 5160-5180

cPPT: 5886-5901

U3PPT: 5886-5907

CMV_immearly_promoter: 239-810

Ubiquitin Promoter: 2617-3833

SV40 promoter: 3964-4341

AmpR_promoter: 10327-10355

pBR322_origin: 8651-9270

Primer locations and sequences

Ubi-F (3756-3778): GGGTCAATATGTAATTTTCAGTG

FLAG-R-1 (3963-3943): GCTAGCTCATTTGTCGTCATC

Carrier Atlas

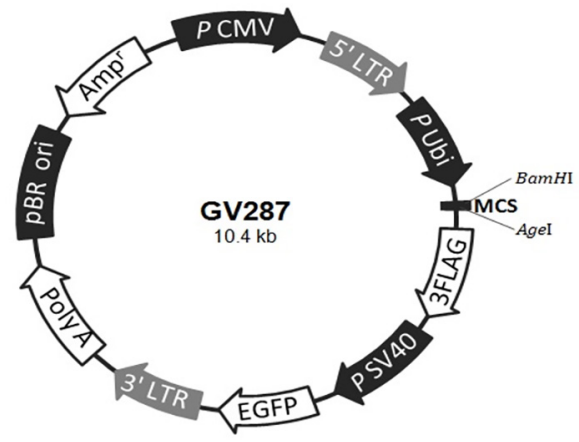


Table S2 Basic information of SNPs located on NFATC1 gene detected by TS based on NGS

\begin{tabular}{|c|c|c|c|c|c|}
\hline Chromosome & Location & Reference allele & Alternative allele & Function & SNP nomination \\
\hline chr18 & 77156171 & $\mathrm{G}$ & $\mathrm{C}$ & UTR5 & rs74183647 \\
\hline chr18 & 77156174 & $\mathrm{G}$ & $A$ & UTR5 & rs139536097 \\
\hline chr18 & 77156197 & $\mathrm{C}$ & $\mathrm{T}$ & UTR5 & rs62096862 \\
\hline chr18 & 77156449 & $\mathrm{G}$ & $\mathrm{C}$ & Intronic & rs58346397 \\
\hline chr18 & 77156458 & A & $G$ & Intronic & rs8097032 \\
\hline chr18 & 77160567 & $\mathrm{C}$ & $\mathrm{G}$ & Intronic & rs560385670 \\
\hline chr18 & 77160601 & $\mathrm{C}$ & A & Intronic & rs62096871 \\
\hline chr18 & 77170558 & $\mathrm{G}$ & $A$ & Exonic & rs781552559 \\
\hline chr18 & 77170776 & C & $\mathrm{T}$ & Exonic & rs768935319 \\
\hline chr18 & 77170857 & $\mathrm{G}$ & A & Exonic & rs199503337 \\
\hline chr18 & 77171061 & $\mathrm{~T}$ & $\mathrm{G}$ & Exonic & rs2230112 \\
\hline chr18 & 77171135 & $\mathrm{C}$ & $\mathrm{T}$ & Exonic & rs200533002 \\
\hline chr18 & 77171164 & $\mathrm{G}$ & $A$ & Exonic & rs76525142 \\
\hline chr18 & 77171171 & $\mathrm{C}$ & $\mathrm{T}$ & Exonic & rs201850664 \\
\hline chr18 & 77171292 & C & $\mathrm{T}$ & Exonic & rs200853741 \\
\hline chr18 & 77171313 & $\mathrm{G}$ & $A$ & Exonic & rs777408035 \\
\hline chr18 & 77171481 & $\mathrm{G}$ & $\mathrm{C}$ & Exonic & rs200146253 \\
\hline chr18 & 77171523 & $\mathrm{G}$ & $A$ & Intronic & rs540117854 \\
\hline chr18 & 77193717 & $\mathrm{C}$ & $\mathrm{T}$ & Exonic & rs184916992 \\
\hline chr18 & 77208693 & $\mathrm{G}$ & A & Intronic & - \\
\hline chr18 & 77208735 & $\mathrm{G}$ & $A$ & Intronic & rs778301024 \\
\hline chr18 & 77208922 & C & $\mathrm{T}$ & Exonic & rs25657 \\
\hline chr18 & 77209015 & $A$ & $\mathrm{G}$ & Intronic & rs2278801 \\
\hline chr18 & 77210902 & C & $\mathrm{T}$ & Intronic & rs680802 \\
\hline chr18 & 77211175 & $\mathrm{~T}$ & $\mathrm{C}$ & Intronic & rs2290154 \\
\hline chr18 & 77211177 & C & $\mathrm{T}$ & Intronic & rs373993229 \\
\hline chr18 & 77211195 & $\mathrm{G}$ & $\mathrm{T}$ & Intronic & rs160191 \\
\hline chr18 & 77211764 & $\mathrm{~T}$ & C & Exonic & rs15350 \\
\hline chr18 & 77211795 & $A$ & $\mathrm{G}$ & Exonic & rs747539233 \\
\hline chr18 & 77211856 & $\mathrm{G}$ & $A$ & Intronic & - \\
\hline chr18 & 77211872 & $\mathrm{G}$ & $A$ & Intronic & rs189757547 \\
\hline chr18 & 77211927 & $A$ & $\mathrm{G}$ & Intronic & rs548161343 \\
\hline chr18 & 77211959 & $\mathrm{G}$ & $\mathrm{T}$ & Intronic & - \\
\hline chr18 & 77221207 & $\mathrm{C}$ & $A$ & Intronic & rs2304738 \\
\hline chr18 & 77221264 & $\mathrm{C}$ & $\mathrm{T}$ & Intronic & rs2304739 \\
\hline chr18 & 77221278 & $\mathrm{G}$ & $A$ & Intronic & rs56112793 \\
\hline chr18 & 77227285 & $\mathrm{~T}$ & C & Intronic & rs2304741 \\
\hline chr18 & 77227476 & $A$ & $\mathrm{G}$ & Exonic & rs25656 \\
\hline chr18 & 77227610 & $\mathrm{G}$ & $A$ & Exonic & rs55740492 \\
\hline chr18 & 77246305 & $\mathrm{C}$ & $\mathrm{T}$ & Exonic & rs774688540 \\
\hline chr18 & 77246406 & $\mathrm{~T}$ & $\mathrm{G}$ & Exonic & rs754093 \\
\hline chr18 & 77246428 & $\mathrm{~T}$ & C & Exonic & rs753386603 \\
\hline chr18 & 77246444 & $\mathrm{C}$ & $\mathrm{T}$ & Exonic & rs7233684 \\
\hline chr18 & 77246527 & C & $\mathrm{T}$ & Exonic & rs200207441 \\
\hline chr18 & 77246538 & $\mathrm{G}$ & $A$ & Exonic & rs56191735 \\
\hline chr18 & 77246548 & C & $\mathrm{T}$ & Exonic & rs756675542 \\
\hline chr18 & 77246589 & $G$ & $A$ & Exonic & rs201484637 \\
\hline chr18 & 77246936 & $\mathrm{C}$ & $\mathrm{T}$ & Exonic & rs150249025 \\
\hline chr18 & 77246977 & $\mathrm{C}$ & $\mathrm{T}$ & Intronic & rs374978531 \\
\hline chr18 & 77246978 & $\mathrm{G}$ & $A$ & Intronic & rs754096 \\
\hline chr18 & 77247051 & $\mathrm{C}$ & $\mathrm{T}$ & Intronic & - \\
\hline chr18 & 77287385 & $\mathrm{~T}$ & $\mathrm{C}$ & Intronic & rs451691 \\
\hline chr18 & 77287440 & $\mathrm{C}$ & $G$ & Intronic & rs535298168 \\
\hline chr18 & 77287561 & $\mathrm{C}$ & $\mathrm{T}$ & Exonic & rs149271669 \\
\hline chr18 & 77287776 & $A$ & $\mathrm{G}$ & UTR3 & rs1052025 \\
\hline
\end{tabular}


Table S3 Results of MAF and HWE analysis of all 55 SNPs detected by the TS on NFATC1 gene based on the genetic

\begin{tabular}{|c|c|c|c|}
\hline SNP & MAF & HWE & REF:ALT \\
\hline rs754096 & 0.487 & 0.2683 & G:A \\
\hline rs2290154 & 0.417 & 0.2167 & $\mathrm{~T}: \mathrm{C}$ \\
\hline rs2278801 & 0.407 & 0.1776 & $A: G$ \\
\hline rs15350 & 0.4 & 0.1114 & $\mathrm{~T}: \mathrm{C}$ \\
\hline rs754093 & 0.395 & 0.8641 & $T: G$ \\
\hline rs25656 & 0.372 & 0.3425 & $A: G$ \\
\hline rs2304738 & 0.367 & 0.1794 & C:A \\
\hline rs2304739 & 0.365 & 0.2174 & C:T \\
\hline rs56112793 & 0.365 & 0.2174 & G:A \\
\hline rs62096871 & 0.3 & $2.47 \mathrm{E}-53$ & C:A \\
\hline rs74183647 & 0.268 & 7.36E-05 & G:C \\
\hline rs2230112 & 0.133 & 0.5831 & G:T \\
\hline rs58346397 & 0.125 & $5.31 \mathrm{E}-33$ & G:C \\
\hline rs62096862 & 0.105 & 0.3049 & C:T \\
\hline rs139536097 & 0.095 & 0.1654 & G:A \\
\hline rs25657 & 0.075 & 1 & C:T \\
\hline rs8097032 & 0.035 & $1.87 \mathrm{E}-13$ & $A: G$ \\
\hline rs560385670 & 0.018 & 1 & $C: G$ \\
\hline rs451691 & 0.013 & $2.00 \mathrm{E}-04$ & $\mathrm{~T}: \mathrm{C}$ \\
\hline rs373993229 & 0.007 & 1 & C:T \\
\hline rs535298168 & 0.007 & 1 & $C: G$ \\
\hline rs201850664 & 0.005 & 1 & $\mathrm{C}: \mathrm{T}$ \\
\hline rs189757547 & 0.005 & 1 & G:A \\
\hline rs548161343 & 0.005 & 1 & $A: G$ \\
\hline rs753386603 & 0.005 & 1 & $\mathrm{~T}: \mathrm{C}$ \\
\hline rs781552559 & 0.003 & 1 & G:A \\
\hline rs768935319 & 0.003 & 1 & C:T \\
\hline rs199503337 & 0.003 & 1 & G:A \\
\hline rs200533002 & 0.003 & 1 & C:T \\
\hline rs76525142 & 0.003 & 1 & G:A \\
\hline rs200853741 & 0.003 & 1 & C:T \\
\hline rs777408035 & 0.003 & 1 & G:A \\
\hline rs200146253 & 0.003 & 1 & G:C \\
\hline rs540117854 & 0.003 & 1 & G:A \\
\hline rs184916992 & 0.003 & 1 & C:T \\
\hline Chr18:77208693 & 0.003 & 1 & G:A \\
\hline rs778301024 & 0.003 & 1 & G:A \\
\hline rs747539233 & 0.003 & 1 & $A: G$ \\
\hline Chr18:77211856 & 0.003 & 1 & G:A \\
\hline Chr18:77211959 & 0.003 & 1 & G:T \\
\hline rs2304741 & 0.003 & 1 & $\mathrm{~T}: \mathrm{C}$ \\
\hline rs55740492 & 0.003 & 1 & G:A \\
\hline rs774688540 & 0.003 & 1 & C:T \\
\hline rs7233684 & 0.003 & 1 & C:T \\
\hline rs200207441 & 0.003 & 1 & C:T \\
\hline rs56191735 & 0.003 & 1 & G:A \\
\hline rs756675542 & 0.003 & 1 & C:T \\
\hline rs201484637 & 0.003 & 1 & G:A \\
\hline rs150249025 & 0.003 & 1 & C:T \\
\hline rs374978531 & 0.003 & 1 & C:T \\
\hline Chr18:77247051 & 0.003 & 1 & C:T \\
\hline rs149271669 & 0.003 & 1 & C:T \\
\hline rs1052025 & 0.003 & 1 & $A: G$ \\
\hline rs680802 & 0 & 1 & $\mathrm{~T}: \mathrm{T}$ \\
\hline rs160191 & 0 & 1 & $\mathrm{~T}: \mathrm{T}$ \\
\hline
\end{tabular}

MAF, major allele frequency; HWE, Hardy-Weinberg equilibrium;

TS, target sequencing; SNPs, single nucleotide polymorphisms. 
Table S4 Results of association analysis of 10 tagger SNPs on NFATC1 gene with post-transplant BPAR episodes using multivariable regression analysis adjusted by CsA/TAC usage and sirolimus administration

\begin{tabular}{|c|c|c|c|c|}
\hline SNPs & Model & OR & $95 \% \mathrm{Cls}$ & $P$ value \\
\hline \multirow[t]{4}{*}{ rs139536097 } & Additive & 0.97 & $0.49-1.91$ & 0.94 \\
\hline & Dominant & 1.03 & $0.47-2.25$ & 0.95 \\
\hline & Recessive & 0.61 & $0.06-6.15$ & 0.67 \\
\hline & HOM & 0.62 & $0.06-6.25$ & 0.68 \\
\hline rs560385670 & Dominant & 0.28 & $0.03-2.41$ & 0.24 \\
\hline \multirow[t]{2}{*}{ rs2230112 } & Additive & 0.81 & $0.42-1.57$ & 0.54 \\
\hline & Dominant & 0.76 & $0.38-1.52$ & 0.43 \\
\hline \multirow[t]{2}{*}{ rs201850664 } & Additive & 1.54 & $0.09-25.96$ & 0.77 \\
\hline & Dominant & 1.54 & $0.09-25.96$ & 0.77 \\
\hline \multirow[t]{3}{*}{ rs25657 } & Additive & 0.69 & $0.29-1.62$ & 0.39 \\
\hline & Dominant & 0.71 & $0.29-1.70$ & 0.44 \\
\hline & HET & 0.74 & $0.31-1.79$ & 0.50 \\
\hline \multirow[t]{2}{*}{ rs373993229 } & Additive & 0.88 & $0.07-10.29$ & 0.92 \\
\hline & Dominant & 0.88 & $0.07-10.29$ & 0.92 \\
\hline rs189757547 & Additive & 1.06 & $0.063-18.06$ & 0.97 \\
\hline \multirow[t]{2}{*}{ rs753386603 } & Additive & $7.43 \mathrm{E}-10$ & - & 0.99 \\
\hline & Dominant & 7.43E-10 & - & 0.99 \\
\hline
\end{tabular}

*, $P$ value less than adjusted $P$ value $(0.05 / 14=0.0036)$ was considered as statistical significant. BPAR, biopsy-proven acute rejection; SNPs, single nucleotide polymorphisms; OR, odds ratio; Cls, confidential intervals; CsA, cyclosporine A; TAC, tacrolimus. 
Table S5 Results of association analysis of three significant tagger SNPs with the pathological scores of 69 recipients in BPAR group using multivariable logistic regression analysis

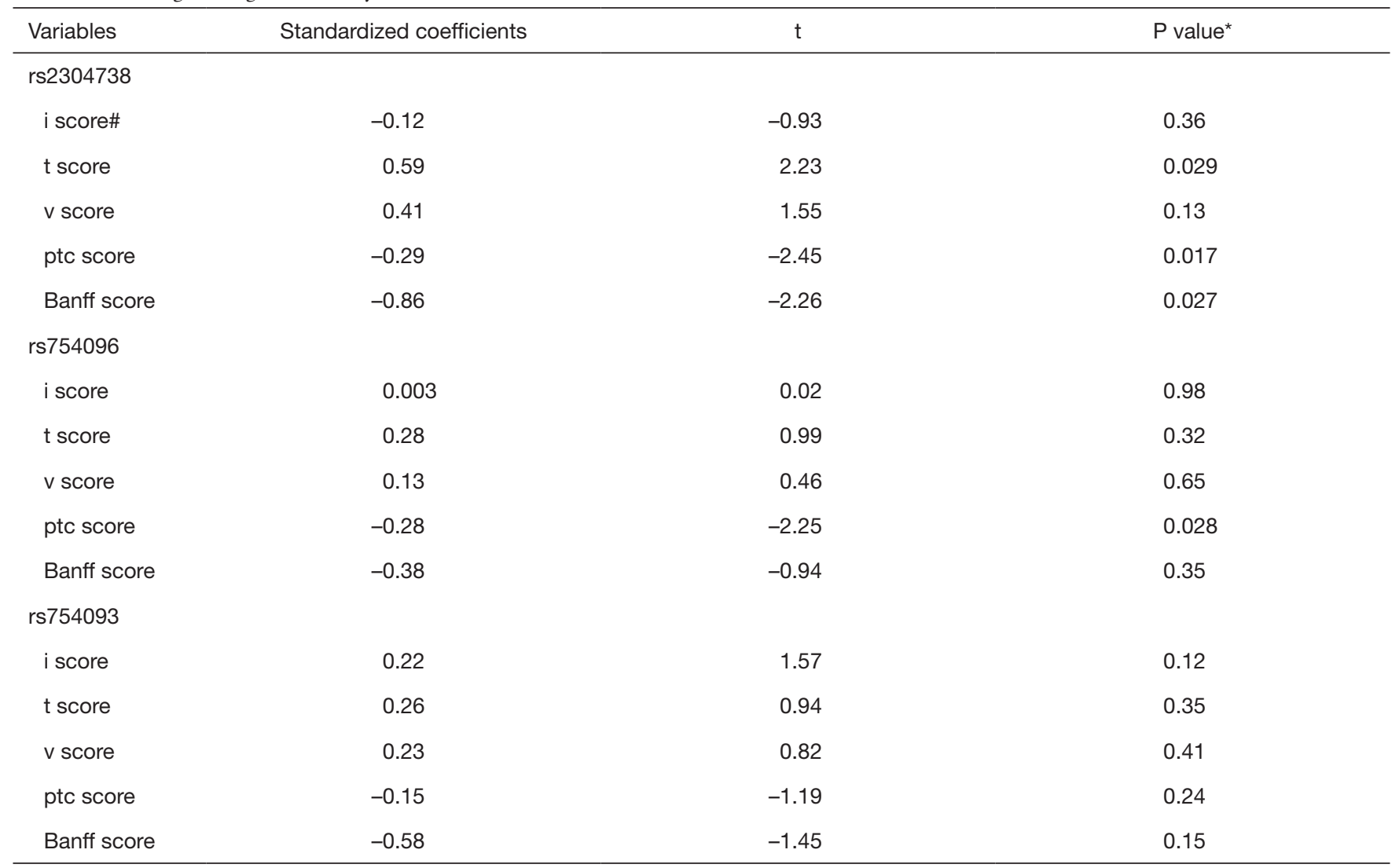

*, $\mathrm{P}$ value less than adjusted $\mathrm{P}$ value $(0.05 / 4=0.0125)$ was considered as statistical significant; , Banff score was obtained according to the Banff 2007 classification. The i, g, t, v, and ptc scores represent the interstitial inflammation, glomerulitis, tubulitis, intimal arteritis, and peritubular capillaritis, respectively. SNPs, single nucleotide polymorphisms; BPAR, biopsy-proven acute rejection. 